\title{
LA AUDITORÍA COMO MEJORA CONTINUA EN LOS ORGANISMOS PÚBLICOS DE SALUD DEL PERÚ
}

\author{
THE AUDIT AS CONTINUOUS IMPROVEMENT IN PERU'S PUBLIC \\ HEALTH AGENCIES
}

Alberto Benjamín Espinoza Valenzuela Universidad Nacional Mayor de San Marcos Lima-Perú

ORCID: https://orcid.org/0000-0001-8577-1717

Correo electrónico: aespinozaoci@gmail.com

\section{RESUMEN}

Objetivo: Describir y explicar cómo la auditoría con criterios de mejora continua puede mejorar la gestión de procesos en las entidades del Estado que prestan servicios asistenciales, teniendo como base las recomendaciones que fluyen de los informes de los órganos de control institucional, que conforman el Sistema Nacional de Control, con el propósito de lograr criterios básicos de eficiencia, eficacia, economía y legalidad en las prestaciones de salud que realiza el sector público, representados por el Ministerio de Salud (Minsa) y el Seguro Social del Perú (EsSalud). Método: El enfoque del estudio es cuantitativo; el tipo de investigación, descriptivo, correlacional y exploratorio, con diseño transaccional no experimental. Se aplicaron cuestionarios en escala de Likert y en escala dicotómica, cuyo análisis se realizó comparando los procedimientos técnicos de auditoría efectuados en los hospitales de Minsa y Essalud, mediante el análisis de casos, encuestas y entrevistas en dichas entidades de salud. Resultados: La prestación de los servicios asistenciales dependen, de alguna manera, de la implementación de recomendaciones provenientes de los servicios de auditoría efectuados, sin la cual los servicios de prestaciones asistenciales continúan siendo deficientes. Conclusiones: Los Sistemas de Control Interno y Auditoría no coadyuvan a la mejora de los sistemas de atención hospitalaria, incrementándose el riesgo que estas deficiencias afecten adversamente al público usuario.

Palabras clave: Auditoría; Minsa; EsSalud; Organismos Públicos.

\begin{abstract}
Objective: The research aimed to describe and explain how audit with criteria for continuous improvement could to improve the management of processes in state entities that provide care services, based on the recommendations that flow from the reports of the institutional control bodies, forming the National Control System, with the aim of achieving basic criteria of efficiency, efficiency, economy and legality in the health benefits made by the public sector, represented by the Ministry of Health -Minsa and EsSalud. Method: The approach of the study is quantitative, the type of descriptive, correlational and exploratory research, with non-experimental transactional design. Questionnaires were applied at Likert scale and dichotomous scale, the analysis of which was carried out comparing the technical audit procedures carried out in the hospitals of Minsa and Essalud, by analyzing cases, surveys and interviews in these health entities. Result: The results revealed that the provision of care services in someway depends on the implementation of recommendations from the audit services made, without which the services of assistance services remain poor. Conclusions: Sand concludes that the Internal Control and Audit Systems do not contribute to the improvement of hospital care systems, increasing the risk that these deficiencies adversely affect the user public.
\end{abstract}

Keywords: Audit; Minsa; EsSalud; public bodies.

(c) Los autores. Este artículo es publicado por la Revista Quipukamayoc, Universidad Nacional Mayor de San Marcos. Este es un artículo de acceso abierto, distribuido bajo los términos de la Licencia Creative Commons Atribución-NoComercial-Compartirlgual 4.0 Internacional.(http://creativecommons.org/licenses/by-nc-sa/4.0/), que permite el uso no comercial, distribución y reproducción en cualquier medio, siempre que la obra original sea debidamente citadas. 


\section{INTRODUCCIÓN}

La investigación busca definir el rol de la auditoría en los organismos públicos de salud en el país, que coadyuve a mejorar los niveles de gestión de las entidades que brindan atención en salud pública en el Perú como son el Ministerio de Salud (Minsa) y el Seguro Social de Salud del Perú (EsSalud), en las cuales las auditorías han emitido informes y en consecuencia recomendaciones, únicamente con fines punitivos de identificación de responsabilidades administrativas y presupuestales, dejando de lado aspectos vinculados a la adecuada prestación de los servicios básicos de salud que en los últimos años se ha constituido en una demanda permanente por parte de la población usuaria.

Para el desarrollo de la investigación se han revisado algunos estudios como el de Barbarán (2015) quien señala que: "La calidad de los informes de auditoría permiten mejorar la toma de decisiones; y, que la Auditoría gubernamental incide positivamente en el desarrollo de la gestión de las entidades públicas en el Perú” (p. 158). Lo señalado muestra la necesidad de adoptar nuevas estrategias de auditoría por parte de los Órganos de Control de las entidades que prestan atención de salud pública, con el fin de recomendar mejoras que coadyuven a la mejor atención de los servicios básicos de salud.

Asimismo, Córdoba (2016) precisa que la "auditoría gubernamental fortalece la gestión de riesgos, maximiza el uso de los recursos de auditoría, agregando valor y fomentando la calidad y las mejores prácticas" (p. 65), que en el caso de los servicios de salud pública se torna fundamental por la importante contribución que el rol de la Auditoría gubernamental asume como valor agregado de su ejecución al señalar que se debe fomentar la calidad y mejores prácticas de atención de los servicios asistenciales, como producto de sus recomendaciones.

Igualmente, Tirado (2017) puntualiza que "el control gubernamental actúa como un facilitador al lograr la eficacia en la implementación de proyectos de inversión en aras de generar resultados que impacten positivamente en el bienestar del ciudadano y en el desarrollo del país" (p. 1), que en el presente contexto define la necesidad de que los resultados de una auditoría no solo analicen los procesos efectuados y/o el producto logrado, como auditoría "posterior" con características "detectivas", sino el impacto de ambas variables en el servicio público que se espera lograr y para los cuales se han ejecutado, es decir, una auditoría "preventiva", de característica "proactiva"

Por ello, la investigación toma como premisa la posición del control gubernamental en el círculo de mejora continua en la organización PHVA (Planificar, Hacer, Verificar y Actuar) para la prestación de servicios públicos de salud, en las etapas de verificar y actuar, buscando demostrar que, en los sectores sociales, preferentemente en el sector salud, todas las auditorías deben tener como finalidad, además de la verificación del uso legal de los recursos públicos, la mejora de la calidad de atención de los pacientes a través del servicio asistencial. Al respecto Zegarra (2010) como corolario de su trabajo de investigación concluye que la implementación de recomendaciones de la Auditoría gubernamental influye en los objetivos institucionales de las entidades públicas (p.93)

Un análisis previo de los informes de auditoría demuestra preliminarmente que existen deficiencias e ineficiencias de los distintos sistemas institucionales de EsSalud y que, pese a las recomendaciones dadas por el Órgano de Control Institucional (OCI), no se han observado mejoras significativas en la calidad de atención asistencial y la insatisfacción interna y externa se mantiene e incrementa.

Igualmente sucede en el Ministerio de Salud en el que, de acuerdo con las estadísticas de acciones de control efectuadas, la calidad de atención a los pacientes del sector no ha sido la más adecuada. A pesar de las recomendaciones para mejorar la gestión institucional del Ministerio de Salud, no se aprecia que los funcionarios responsables de aplicar los correctivos necesarios hayan dispuesto lo necesario para implementar tales recomendaciones. Es decir, existe un vacío entre el nivel de recomendaciones emitidas y su implementación. Según Betancourt (2006) “en la gestión por resultados los directivos tienen el compromiso de conducir hacia el logro de la eficiencia, efectividad, eficacia, calidad y satisfacción de la población, para la consecución de los objetivos de política y mejora continua de sus procesos" (p. 7).

Además de ello, la falta de decisión para implementar el Sistema de Control Interno Institucional dispuesto por la Ley $\mathrm{N}^{\circ} 28716$, así como por las Resoluciones de la Contraloría General de la República $N^{\circ} 320-\mathrm{CG}-06$ y Nº 458CG-08 que disponen la implantación de un modelo de Control Interno a partir del modelo COSO (Committee of Sponsoring Organizations of the Treadway) (Mantilla, 2003), en el cual la planificación y gestión institucional deben concatenar adecuadamente los principios de riesgo y actividades de control, de forma tal que aseguren el cumplimiento de los objetivos institucionales.

En este extremo del diagnóstico efectuado, se toma como orientación, las hipótesis siguientes:

1. El Ministerio de Salud y Essalud, se encuentran debidamente organizados en un ambiente de control adecuado. 
2. En estas instituciones, las auditorías que se practican tienen un sesgo preferencial hacia un control reactivo-posterior con alcances retrospectivos, en detrimento de un rol proactivo con alcances prospectivos.

3. La no aplicación de procedimientos de auditoría que evalúen la calidad de los servicios asistenciales disminuye la oportunidad del control y auditoría gubernamental en relación con su efectividad en la cautela y buen uso de los recursos públicos.

\section{MATERIALES Y MÉTODOS}

La investigación es descriptiva, correlacional y de diseño no experimental. Este tipo de investigación se realizó en base al estudio, análisis y descripción de las situaciones relativas a las auditorías y sus niveles de calidad, es decir, se describe el problema de estudio sin que se realice ninguna modificación. Es un trabajo longitudinal porque se han analizado las variables interrelacionadas en un periodo de tiempo, (Hernández, 2014), de 2016 al 2017. Finalmente constó de dos campos, una documental y una de campo.

En la investigación, se considera como dimensión espacial el análisis de los Órganos de Control Institucional tanto del Minsa como de EsSalud en base a criterios de materialidad poblacional (porcentaje de población adscrita a sus servicios); así como al volumen presupuestal que se les asigna, considerando como Dimensión Temporal para la investigación los años 2016 y 2017. Una muestra con criterio discrecional e intencional donde se seleccionó los elementos que a juicio de la investigación son representativos, relevantes por conocimiento previo de la investigación, determinando los elementos que se consideraron como representativos del fenómeno que se estudia (Sabino, 1992; Tamayo. 1999).

En este sentido para el levantamiento de información, se tomó en consideración las sedes administrativas y asistenciales a nivel central y a nivel descentralizado en los principales hospitales del Minsa y EsSalud. La muestra tomada fue de 150 personas, entre trabajadores de los sistemas de control y gestión, así como asistenciales.

En la investigación se usó el análisis documental y la encuesta como la principal herramienta de recolección de datos, aplicando técnicas de observación, entrevistas y análisis documental. La encuesta posibilitó obtener información de manera efectiva y eficiente las cuales fueron analizadas convenientemente. De esta manera los instrumentos fueron los medios materiales que se emplean para recoger y almacenar información Arias (2012).

En cuanto al análisis e interpretación de la información, se empleó el análisis documental llegando a revisar la documentación minuciosa que se relaciona con el problema; la tabulación de encuestas, información obtenida del personal de auditoría y de los principales funcionarios de ambas instituciones públicas asistenciales (Peña y Pirela, 2007); .Finalmente, en el procedimiento estadístico se empleó, principalmente, el software SPSS, que posibilitó determinar los porcentajes y frecuencias de salida de cada una de las categorías en las respuestas obtenidas mediante encuestas.

Para llevar a cabo este análisis estadístico se utilizó como instrumento el análisis de correlación entre las variables. Principalmente el coeficiente de Correlación de Pearson, técnica bivariada que permite establecer similitudes o disimilitudes entre las variables (Restrepo y Gonzáles, 2007).

Los procedimientos empleados en la investigación fueron los siguientes:

1. Se recolectó información de los Órganos de Control Institucional (OCI) del Minsa y de ESSALUD así como de los representantes de funcionarios de ambas instituciones. Igualmente se recopiló información de los representantes asistenciales de los principales hospitales de ambas instituciones.

2. Se analizó información y se clasificó respecto a los objetivos y fines de las auditorías y las implicancias y resultados de cada uno de ellos para mejorar los servicios médicos asistenciales en cada dependencia.

3. Se trabajó analíticamente mediante las tabulaciones de las respuestas a nivel de cada pregunta a fin de efectuar su contrastación y se procesó mediante el estadístico SPSS a fin de establecer la correlación de las variables y determinar si son independientes o dependientes entre sí.

\section{RESULTADOS}

Para la primera hipótesis: "El Ministerio de Salud y Essalud, se encuentran debidamente organizados en un ambiente de control adecuado", de un total de 150 encuestados, debidamente contrastados con la documentación que sustenta las respuestas se obtuvo los siguientes resultados (Ver Tabla 1).

De la Tabla 1, se desprende que no hay un ambiente de control interno operativo administrativo hasta en un 58\%, considerándose los componentes del Sistema de Control Interno Público aprobado por Ley $\mathrm{N}^{\circ} 28716$, con una ausencia de adecuada organización, niveles de planificación, estrategias organizativas, manejo de recursos humanos y vigencia de documentos normativos. Los resultados, además, demostraron un $63 \%$ de ausencia de implementación de ambiente de control en las entidades del Minsa y EsSalud. 
Igualmente, no se percibe una política de evaluación de riesgos que prime la identificación de eventos negativos, estimación de probabilidades e impacto de riesgos, así como políticas de respuesta al riesgo, que minimicen estas contingencias, pues el análisis efectuado nos arroja un $59 \%$ de ausencia de evaluación de riesgo. En cuanto a la práctica de actividades de información y comunicación (62\%) y actividades de control gerencial (37\%), es notoria la falta de políticas de control preventivas, detectivas y correctivas en estas entidades que minimiza la práctica de controles internos señalada.

Finalmente se aprecia que la ausencia de la supervisión y monitoreo es alta, llegando al 69\%; que correlacionada con la ausencia de control en las prestaciones médicas de hasta un $79 \%$, indica la dependencia que hay entre la ausencia de control operativo y administrativo en estas entidades y la prestación de los servicios asistenciales.

En cuanto a la segunda hipótesis: "En el Ministerio de Salud y ESSALUD, las auditorías que se practican tienen un sesgo preferencial hacia un control reactivo-posterior con alcances retrospectivos, en detrimento de un rol proactivo con alcances prospectivos", se percibe que la intervención de la Auditoría gubernamental por parte de los órganos de control institucional y/o de auditoría no prestan una intervención eficaz en pro de los servicios asistenciales, a pesar de que las jefaturas y/o gerencias tratan de prestar la debida atención a la implementación de sus recomendaciones (según la Tabla 2 hasta en un $80 \%$ ).

En la Tabla 2 también se aprecia los bajos índices de respuesta que tiene la función de Auditoría gubernamental para cumplir con sus responsabilidades, la misma que se ve reflejada por la no disponibilidad de información documentada fiable para realizar sus funciones.

Esta situación es corroborada por información en los propios órganos de control institucional que tienen limitaciones en el alcance de sus auditorías, ya que el sistema actual no les permite abordar temas asistenciales, sino únicamente auditorías detectivas de carácter posterior en temas administrativo- operativos.

Del mismo modo, para el análisis de la segunda hipótesis se ha evidenciado, según la Tabla 3 , que la auditoría

Tabla 1

Ambiente de Control y Prestación de Servicios Médicos

\begin{tabular}{lc}
\hline & Ausencia de control en las prestaciones médicas \\
\hline Ausencia de Ambiente de Control operativo administrativo & $58 \%$ \\
Implementación de ambiente de control & $63 \%$ \\
Evaluación de riesgos & $59 \%$ \\
Actividades de información y comunicación & $62 \%$ \\
Actividades de control gerencial & $37 \%$ \\
Monitoreo de control interno & $69 \%$ \\
Ausencia de Control en Prestaciones Médicas & \\
\hline
\end{tabular}

Fuente: Elaboración propia (2017)

Tabla 2

Auditoría gubernamental en temas asistenciales

\begin{tabular}{|c|c|c|c|c|}
\hline \multirow{2}{*}{ Nivel de intervención de Auditoría gubernamental } & \multicolumn{4}{|c|}{ Función sustantiva } \\
\hline & ALTO & MODERADO & BAJO & MUY BAJO \\
\hline Existe una intervención eficaz por parte del OCl & $11 \%$ & $19 \%$ & $47 \%$ & $23 \%$ \\
\hline $\begin{array}{l}\text { Las jefaturas/gerencias prestan la atención apropiada a la implementa- } \\
\text { ción de recomendaciones. }\end{array}$ & $10 \%$ & $70 \%$ & $12 \%$ & $8 \%$ \\
\hline $\begin{array}{l}\text { EI OCI recibe suficiente información que le permita cumplir con sus } \\
\text { responsabilidades }\end{array}$ & $4 \%$ & $15 \%$ & $70 \%$ & $11 \%$ \\
\hline $\begin{array}{l}\text { El OCl dispone de información documentaria fiable para realizar las } \\
\text { auditorías }\end{array}$ & $2 \%$ & $8 \%$ & $79 \%$ & $11 \%$ \\
\hline
\end{tabular}

Fuente: Elaboración propia (2017) 
detectiva (posterior) prima sobre la auditoría proactiva (preventiva), convirtiéndose en una seria limitación para el valor agregado que se puede desprender de la Auditoría gubernamental como mejora continua de los servicios asistenciales.

Asimismo, de la información recabada se aprecia el alto porcentaje (73\%) respecto a la necesidad de que el valor agregado de las auditorías sea sustantivo a los temas asistenciales, por lo que los funcionarios perciben la necesidad del control posterior, pero con fines y objetivos de mejora en la gestión. Además, se aprecia un alto porcentaje (98\%) de reconocimiento al control gubernamental como promotor de mejora continua o reformas constructivas en materia asistencial.

Por último, se estima que la función auditora debe tener un rol preventivo hacia el logro de resultados, lo que, en la contrastación de las auditorías efectuadas en los años materia de análisis, no ha sido la práctica usual en las emisiones de los informes de control (Ver Tabla 4).

Para el análisis de la tercera hipótesis: "La no aplicación de procedimientos de auditoría que evalúen la calidad de los servicios asistenciales disminuye la oportunidad del control y auditoría gubernamental en relación con su efectividad en la cautela y buen uso de los recursos públicos", los resultados revelan que, en el MINSA y EsSalud, los Órganos de Control Institucional (OCI) no evalúan el riesgo integral (79\%). Asimismo, de acuerdo con un $84 \%$ de los encuestados, debe profundizarse las investigaciones en los hallazgos con el impacto relativo a la calidad asistencial que brinda la entidad. Además, se observa un $81 \%$ de aprobación para considerar en el efecto de auditoría el impacto en el servicio que se brinda al usuario.

Igualmente, se aprecia que el $88 \%$ de los encuestados prefieren un esquema de programación de auditoría que relacione la ejecución presupuestal, es decir, el gasto con el nivel de servicio que se brinda a la entidad, respuesta concordante con que debe relacionarse la causa que genera el hallazgo considerando el servicio final al usuario, las respuestas afirmativas para esta pregunta tuvieron un $74 \%$. Finalmente, se concluye que el control preventivo debe tener características de prevención antes que detección, enfocándose a mejorar la calidad del servicio, como fin último de las entidades que brindan servicios de salud, alcanzando un $85 \%$ de aceptación.

Tabla 3

El órgano de control y los servicios asistenciales

\begin{tabular}{|c|c|c|c|c|}
\hline \multirow[b]{2}{*}{ Atención del $\mathrm{OCl}$ en temas asistenciales } & \multicolumn{4}{|c|}{ Función sustantiva } \\
\hline & $\begin{array}{l}\text { Totalmente } \\
\text { de acuerdo }\end{array}$ & De acuerdo & $\begin{array}{c}\text { En } \\
\text { desacuerdo }\end{array}$ & $\begin{array}{c}\text { Totalmente en } \\
\text { desacuerdo }\end{array}$ \\
\hline Auditoría detectiva prima sobre la auditoría proactiva & $1 \%$ & $24 \%$ & $64 \%$ & $11 \%$ \\
\hline Debe existir mayor valor agregado de auditoría en temas asistenciales & $6 \%$ & $67 \%$ & $14 \%$ & $13 \%$ \\
\hline La Auditoría gubernamental como herramienta de mejora continua & $9 \%$ & $89 \%$ & $2 \%$ & $0 \%$ \\
\hline Control preventivo y logro de resultados & $16 \%$ & $72 \%$ & $10 \%$ & $2 \%$ \\
\hline
\end{tabular}

Fuente: Elaboración propia (2017)

Tabla 4

Impacto de la Auditoría en temas asistenciales

\begin{tabular}{|c|c|c|}
\hline \multirow{2}{*}{ Auditoría como mejora continua de temas asistenciales } & \multicolumn{2}{|c|}{ Vigencia } \\
\hline & AFIRMATIVO & NEGATIVO \\
\hline Se efectúa una evaluación de riesgo integral en la planificación de auditorías asistenciales & $32(21 \%)$ & $118(79 \%)$ \\
\hline En las auditorías deben profundizarse los impactos asistenciales & $126(84 \%)$ & $24(16 \%)$ \\
\hline Debe considerarse en el efecto de auditoría el impacto del servicio asistencial que se brinda & $121(81 \%)$ & $29(19 \%)$ \\
\hline La auditoría debe profundizar evaluar los insumos asistenciales utilizados & $132(88 \%)$ & $18(12 \%)$ \\
\hline $\begin{array}{l}\text { La auditoría debe profundizar la causa que genera el hallazgo, considerando el servicio final que se } \\
\text { brinde al usuario }\end{array}$ & $111(74 \%)$ & $39(26 \%)$ \\
\hline $\begin{array}{l}\text { Es mejor hacer un Control Preventivo conjunto de Control de recursos enfocado a mejorar el servicio } \\
\text { de la entidad. }\end{array}$ & $128(85 \%)$ & $22(15 \%)$ \\
\hline
\end{tabular}

Fuente: Elaboración propia (2017) 
En la tabla 5 se muestra que el valor de correlación de Pearson significativo (p.sig= bilateral) para las hipótesis planteadas a un nivel de significancia de $\alpha=0,05$ son menores y que los resultados del coeficiente de correlación entre las variables planteadas son bastante buenas $r=a l$ menos 0,667 por lo que, en todos los casos, se rechaza la hipótesis nula y se aceptan las hipótesis alternas.

Sin embargo, a pesar del alto grado de correlación que se muestra, los resultados respecto a la organización y gestión en instituciones que brindan servicios de salud pública y las causas que limitan un sistema de control interno favorable en las prestaciones médicas, muestran resultados preocupantes (79\%) que generan ausencia de control interno en las prestaciones médicas. Situación que se genera por una ausencia visible de ambiente de control operativo administrativo en un 58\% según el relevamiento de datos obtenido

En cuanto a la ausencia de un proceso de control gubernamental específico para temas asistenciales si bien los niveles de correlación también son positivos en 0,923 y 0,903 con un (p.sig= bilateral) de 0,001 y 0,000 menores al nivel de significancia 0,05 lo que hace que se rechace la hipótesis nula y se acepte la hipótesis alterna; esta situación muestra serias limitaciones para el OCI en su intervención en temas asistenciales, imposibilitando que la auditoría gubernamental actúe como herramienta de mejora continua en temas asistenciales.

En relación a la realización de auditorías a la calidad de los servicios asistenciales igualmente la correlación es positiva
0,878 con un $\mathrm{p}$ (p.sig= bilateral) de 0,001 muy por debajo de 0,05 por lo que se rechaza la hipótesis nula y se acepta la hipótesis alterna, lo que demuestra la alta dependencia de efectuar auditorías a la calidad de las prestaciones médicas para que estas alcancen niveles adecuados de calidad. Sobre todo, que las auditorías deben enfocarse a impactos asistenciales en lugar solo de procedimientos administrativos (84\%). Así como enfocarse a evaluar los insumos médicos utilizados (88\%).

\section{DISCUSIÓN}

Este trabajo de investigación concluye que en el Ministerio de Salud y en EsSalud, el Control y Auditoría gubernamental actuales no se efectúan en un ambiente que responda a las necesidades de mejora de los servicios de salud que brindan. Bajo estas consideraciones, el accionar del control interno por parte de la gestión y la Auditoría gubernamental por parte de los Órganos de Control Institucional, vienen teniendo casi nulo efecto en la mejora de los procesos operativos asistenciales, así como en la mejora de la calidad en los servicios médicos que se brinden a los usuarios. Como Davies y Shellard (1997) señalan que los recientes cambios en la perspectiva de gobierno exigen que las auditorías públicas extiendan su ámbito de investigación y análisis en aspectos propios de gestión. Como conclusión del estudio se propone un control gubernamental moderno y de avanzada que no busque únicamente determinar responsabilidades administrativas, civiles o penales; por cuanto se entiende que el fin constitucional del Sistema Nacional de Control es y debe ser coadyuvar

Tabla 5

Relación bivariada entre la Auditoría gubernamental como mejora continua y la mejora de los servicios asistenciales públicos.

\begin{tabular}{|c|c|c|}
\hline \multirow{2}{*}{$\begin{array}{l}\text { Control Gubernamental } \\
\text { Como mejora continua }\end{array}$} & \multicolumn{2}{|c|}{ Mejora de los Servicios Asistenciales Públicos } \\
\hline & Rho de Pearson & Significancia $(p=0.05)$ \\
\hline \multicolumn{3}{|l|}{$1^{\circ}$ Hipótesis. } \\
\hline \multicolumn{3}{|l|}{$\begin{array}{l}\text { Organización y sistema de control interno favorable en las } \\
\text { prestaciones médicas }\end{array}$} \\
\hline - Ambiente de Control & 0,733 & 0,001 \\
\hline - $\quad$ Evaluación de Riesgos & 0,667 & 0,000 \\
\hline - Información y Comunicación & 0,668 & 0,000 \\
\hline - $\quad$ Actividades de Control gerencial & 0,883 & 0,001 \\
\hline - $\quad$ Supervisión y monitoreo & 0,873 & 0,000 \\
\hline \multicolumn{3}{|l|}{$2^{\circ}$ Hipótesis } \\
\hline \multicolumn{3}{|l|}{ Control gubernamental proactivo con alcances prospectivos. } \\
\hline \multirow{3}{*}{$\begin{array}{l}\text { - } \quad \text { Limitaciones para la Intervención del Órgano de Control } \\
\text { Institucional en temas Asistenciales }\end{array}$} & 0,923 & 0.001 \\
\hline & & \\
\hline & 0,903 & 0,000 \\
\hline \multicolumn{3}{|l|}{$3^{\circ}$ Hipótesis } \\
\hline$\overline{\text { Auditoría a la calidad del servicio asistencial }}$ & 0,878 & 0,000 \\
\hline
\end{tabular}

Fuente: Elaboración propia. SPSS (2017) 
al uso adecuado de los recursos públicos en un marco de eficiencia, eficacia y economía con impacto en el servicio público que se brinda al usuario que para el caso de estudio es la mejora continua en la atención de los servicios asistenciales a la población, tal como la Organización Internacional de Entidades Fiscalizadoras Superiores (INTOSAI) organismo representante de las contralorías a nivel Mundial, señala que a las Entidades Fiscalizadoras Superiores, vale decir Contralorías, se les está pidiendo que incorporen una fase de evaluación de impacto dentro de sus tareas de auditoría, a fin de evaluar con mayor precisión la gestión del gobierno (Khemakhe, 2001). De ese modo, pueden suplir una información objetiva y precisa que pueden usar, en primer lugar, los gerentes, y luego las demás autoridades públicas y los ciudadanos.

\section{REFERENCIAS}

Arias, F. (2012). El Proyecto de Investigación. Introducción a la Metodología Científica ( $6^{\mathrm{a}}$ ed.). Caracas: Episteme.

Barbarán, G. (2015). La auditoría gubernamental y su incidencia en el desarrollo de la gestión de las entidades públicas en el Perú (Tesis de doctorado). Universidad San Martín de Porres.

Betancourt, J. (2006). Toma de decisiones: Obtener el éxito. Cuba.

Congreso de la República. (18 de abril de 2006). Ley $\mathrm{N}^{\circ}$ 28716. Ley de Control Interno de las Entidades del Estado.

Contraloría General de la República. (3 de noviembre de 2006). Resolución de Contraloría N³20-CG-06 Normas de Control Interno.

Contraloría General de la República. (30 de octubre de 2008). Resolución de Contraloría $\mathrm{N}^{\circ}$ 458-CG-08 "Guía para la Implementación del Sistema Nacional de Control.

Córdoba, N. (2016). Estrategias de la auditoría gubernamental para erradicar la corrupción en los Municipios distrital de la Provincia de Trujillo (Tesis de maestría). Universidad Nacional de Trujillo.
Mantilla, S. (2003). Control Interno. Informe COSO (3 ed.). Bogotá: ECOE Ediciones.

Davies, M. y Shellard, E. (1997). The Value of Performance Measurement in the United Kingdom. The Government Accountant Journal, 46(3), 48-51.

Hernández, R., Fernández, C., y Baptista, P. (2014) Metodología de la Investigación. México D.F.: McGraw-Hill Interamericana.

Khemakhe, J. (2001). Auditoría Integral. Revista Internacional de Auditoría Gubernamental, 28(2). Recuperado de: https://www.intosai.org/fileadmin/downloads/about_us/IJGA_Issues/former_years/2001/ span_2001_april.pdf

Peña, T. y Pirela, J. (2007). La complejidad del análisis documental. Información, cultura y sociedad, (16), 55-81.

Restrepo, L. y González, J. (2007). De Pearson a Spearman. Revista Colombiana de Ciencias Pecuarias, 20(2), 183-192.

Sabino, C. (1992) El proceso de Investigación. Buenos Aires. Recuperado de: http://paginas.ufm.edu/sabino/ ingles/book/proceso_investigacion.pdf

Tamayo, M. (1999). Módulo 5. El Proyecto de Investigación. Bogotá: Instituto Colombiano para el Fomento de la Educación Superior.

Tirado, K. (2017). Rol de la Contraloría General de la República en proyectos de inversión mediante asociaciones público-privadas (Tesis de maestría). Universidad de Lima.

Zegarra, K. (2010). La implementación de recomendaciones de la auditoría gubernamental y el cumplimiento de los objetivos institucionales de las municipalidades distritales de la ciudad de Tacna, periodo 2007-2009 (Tesis de maestría). Universidad Nacional Jorge Basadre Grohman de Tacna. 
